\title{
Ferramentas de Gestão de Contratos de Serviços de Tecnologia da Informação: análise de aderência à legislação brasileira
}

\author{
Augusto S. C. Modesto ${ }^{1,2}$, Emilie T. de Morais ${ }^{1,2}$, Eduardo H. F. Moreira ${ }^{1}$, Hugo F. \\ Martins $^{1}$, Leonardo C. Corrêa ${ }^{1}$, Rejane M. C. Figueiredo ${ }^{1,2}$ \\ ${ }^{1}$ Universidade de Brasília - Faculdade do Gama, Gama, Distrito Federal, Brasil \\ ${ }^{2}$ ITRAC - Information Technology, Research and Application Center \\ \{augusto.modestoo, emiliemoraist, hugomartins013, leoccambraia, \\ duduhfm\} @gmail.com, rejanecosta@unb.br
}

Abstract. The hiring of information technology (IT) services in Brazilian Federal departments is a complex reality that requires to the departments management policies. At this context. it is essential to employ tools that can support the correct hiring management. Recent guidelines from the Brazilian Federal Government forbids the hiring of software factories services to develop systems that is not related to the main goal of the department. This paper proposes a checklist in order to ensure the agreement of hiring management tools to the norms and guidelines in effect. The checklist was applied on management tools available at Brazilian Public Software Portal. The study of this paper was made in four stages: checklist development, selection of management tools available at Brazilian Public Software Portal, selected management tools evaluation, and reporting of results. The result of the study at this paper concludes that are no IT hiring management tools available at Brazilian Public Software Portal which fulfill the norms and guidelines in effect.

Resumo. A contratação de serviços de Tecnologia da Informação (TI) pelos órgãos públicos federais brasileiros é uma realidade complexa e requer dos órgãos que esses executem a gestão. Nesse cenário é essencial a utilização de um apoio ferramental que possa auxiliar a gerência da execução do contrato. Recentes diretrizes do governo federal vedam a contratação de fábricas para o desenvolvimento de softwares para atividades meio. Este trabalho tem como objetivo a proposição de um checklist para analisar a adequação das ferramentas de gestão de contratos de acordo com as normas e modelos vigentes. O checklist foi aplicado às ferramentas disponiveis no Portal do Software Público Brasileiro (PSPB). Este estudo foi realizado em quatro fases: desenvolvimento do checklist, a seleção das ferramentas disponíveis no PSPB nas categorias que envolvem gestão, a avaliação das ferramentas selecionadas; e a análise e redação dos resultados. Como resultado, constatou-se que as ferramentas disponíveis no PSPB não atendem às normas e modelos vigentes para a contratação de serviços de TI. 


\section{Introdução}

A contratação de serviços de tecnologia da informação (TI) pela Administração Pública Federal (APF) brasileira é prevista em lei [BRASIL 1993], e está descrita no Decreto Lei $n^{\circ}$ 200/67 [BRASIL 1967], que a APF deve desobrigar-se da realização material de tarefas executivas, recorrendo, sempre que possível, à terceirização, mediante contrato.

O Tribunal de Contas da União (TCU) ressaltou a necessidade de cumprimento do princípio constitucional da eficiência e as disposições contidas no art. $6^{\circ}$, I, do Decreto-Lei $n^{\circ} 200 / 67$, criando um processo de planejamento que organizasse as estratégias, as ações, os prazos, os recursos financeiros, humanos e materiais, para que se buscasse eliminar a possibilidade de desperdício de recursos públicos e de prejuízo ao cumprimento dos objetivos institucionais da unidade.

Com a detecção de irregularidades e improbidades no processo de contratação de serviços de TI, O TCU recomendou a Secretaria de Logística e Tecnologia da Informação (SLTI) do Ministério do Planejamento, Orçamento e Gestão (MPOG) que desenvolvesse um modelo de licitação e contratação de serviços de informática e orientasse, normativamente, os órgãos e as entidades da Administração Pública Federal em sua implementação [BRASIL 2003]

Segundo o acórdão 1.603/2008-Plenário TCU [BRASIL 2008], a recomendação foi que o MPOG promovesse "....ações com o objetivo de disseminar a importância do planejamento estratégico, procedendo, inclusive mediante orientação normativa, à execução de ações voltadas à implantação e/ou aperfeiçoamento de planejamento estratégico institucional, planejamento estratégico de TI e comitê diretivo de TI, com vistas a propiciar a alocação dos recursos públicos conforme as necessidades e prioridades da organização...”.

O Ministério do Planejamento, Orçamento e Gestão publicou a Instrução Normativa, Número 04 (IN04) em 2008, versão vigente publicada em 2014 [BRASIL 2014]. A IN04 preconiza que a contratação de cada serviço de tecnologia da informação deve ser oriunda do planejamento estratégico da TI do órgão, e esse, do planejamento estratégico do órgão. As diretrizes para contratação de serviços são organizadas em módulos: o planejamento da contratação; a seleção do fornecedor; e a gestão da contratação. A gestão do contrato compreende a execução dos termos definidos no edital de contratação e busca-se o alcance dos resultados pretendidos com a contratação.

Em paralelo, pesquisas na área contribuíram para definir processos de contratação alinhados à legislação vigente [CRUZ 2008; BRASIL 2009; CRUZ, ANDRADE, FIGUEIREDO 2010], seguidos pela elaboração do Guia Prático de Contratações de TI [BRASIL 2011] pelo MPOG; o Guia de Boas Práticas em Contratação de Solução de TI [BRASIL 2012], pelo Tribunal de Contas da União (TCU), e recentemente, a publicação de Daud Júnior e Vaz [2016].

Os órgãos devem seguir as diretrizes da IN04 [BRASIL 2014] para contratação de serviços de TI, entre eles, a contratação de fábricas de software. As tarefas-meio devem ser realizadas com a qualidade exigida pelo órgão, que deve executar a gestão do contrato, com o controle da eficácia e eficiência previstas no contrato. $\mathrm{O}$ acompanhamento da prestação de serviços dos fornecedores é complexo e demanda sistemas de informação que envolvem desde um pedido de serviço, sua execução, controle da qualidade, a partir dos níveis de serviços (SLA - Service Level Agreements) 
estabelecidos em edital, aceitação ou não das entregas e suas consequências, efetivação do pagamento, e a implantação das entregas no órgão.

Ainda nessa linha, na recém-publicada Portaria $N^{o}$ 20, de 14 de junho de 2016, (DOU de 15/06/2016 (nº 113, Seção 1, pág. 52)), o Secretário a TI do MPOG resolve que as contratações de soluções de Tecnologia da Informação (TI) pelos órgãos e entidades integrantes do Sistema de Administração dos Recursos de Tecnologia da Informação (SISP) devem, entre outras diretrizes, ser precedidas pela análise da:

Equipe de Planejamento da Contratação ou da Equipe de Gestão de Projetos do órgão que deve realizar Estudo Técnico Preliminar, nos termos do disposto no art. 12 da Instrução Normativa SLTI/MP $n^{\circ} 4$, de 11 de setembro de 2014, e executar as seguintes atividades: 1.1. Analisar a existência e a viabilidade de adoção de software que atenda às necessidades da área requisitante no Portal do Software Público Brasileiro (https://softwarepublico.gov.br/);" (Portaria $\left.M P O G / S T I n^{\circ} 20,2016\right)$ [BRASIL 2016].

O Portal do Software Público Brasileiro (PSPB) é um ambiente virtual público criado em 12 de abril de 2007, que disponibiliza soluções de software em diferentes categorias. As soluções têm sua utilização e modificação livres por qualquer cidadão. $\mathrm{O}$ Software Público Brasileiro é um tipo específico de software que adota um modelo de licença livre para o código-fonte, a proteção da identidade original entre o seu nome, marca, código-fonte, documentação e outros artefatos relacionados por meio do modelo de Licença Pública de Marca - LPM. O objetivo é que o Portal se torne um centro de compartilhamento de software, e que possibilite o compartilhamento de soluções pela comunidade e economia de gastos [BRASIL 2016].

Com isso, as recentes diretrizes do governo federal vedam a contratação de fábricas para o desenvolvimento de softwares para atividades meio, entre elas, sistemas de gestão ou administração operacional.

Dado o cenário, o objetivo deste trabalho foi a proposição de um checklist para analisar a adequação das ferramentas de gestão de contratos de TI de acordo com as normas e modelos vigentes. O checklist foi aplicado às ferramentas disponíveis no Portal do Software Público Brasileiro (PSPB).

Este estudo foi realizado em quatro fases: desenvolvimento do checklist, seleção das ferramentas/soluções disponíveis no PSPB nas categorias que envolvem gestão, avaliação das ferramentas selecionadas; e análise e redação dos resultados.

Este trabalho está organizado em seções. Na Seção 2 apresenta-se o referencial teórico com as características da contratação de serviços de TI pela APF e as principais fontes sobre os elementos essenciais e desejáveis à gestão de contratos de TI. Na Seção 3 apresenta-se a metodologia adotada para geração do checklist. Na Seção 4, o desenvolvimento do checklist. Na Seção 5, a seleção das ferramentas do PSPB. Na Seção 6 apresenta-se a análise dos resultados. Na Seção 7, as Considerações Finais.

\section{Referencial Teórico}

Em 2008, Cruz (2008) propôs um Quadro Referencial Normativo (QRN) que alinhava a legislação brasileira vigente (leis, acórdãos, jurisprudência) às fases, atividades e tarefas de um processo de contratação de serviços de TI. Uma das fases previstas no QRN é a 
Gestão Contratual de Serviços de TI, e essa é subdividida em atividades, e essas em tarefas. Com as atividades e tarefas, busca-se o entendimento comum do contrato assinado, o monitoramento técnico e administrativo de sua realização e a transição contratual para garantir a continuidade do serviço prestado [CRUZ 2008].

A IN 04, atualmente na terceira edição, de 2014 [BRASIL 2014], estrutura a contratação em três fases: Planejamento da Contratação, Seleção de Fornecedores e Gestão do Contrato. Tal instrução normativa foi concebida pelo MPOG em sua busca por estabelecer diretrizes para as contratações de TI na APF, respondendo aos acórdãos do TCU em 2008.

O acompanhamento da execução do contrato por parte dos gestores públicos é indicado como uma obrigatoriedade e cabe à fase de Gestão de Contratos realizar um perfeito cumprimento da prestação de serviço durante a vigência do contrato [DAUD JÚNIOR AND VAZ 2016]

Segundo a IN 04 [BRASIL 2014], a fase que trata da gestão do contrato visa conduzir a garantia de que o serviço prestado e os bens fornecidos que compõem a solução de TI estejam de acordo com o solicitado pelo contratante durante todo o período da execução do contrato. Para realização da gestão do contrato são previstas atividades do início do contrato, do encaminhamento formal de demandas, do monitoramento da execução e da transição e, do encerramento contratual.

A partir da IN 04 surgiram outras publicações. Cruz, Andrade e Figueiredo (2010) descrevem um Processo de Contratação de Serviços de TI (PCSTI) para organizações públicas, composto de quatro fases: Planejamento de TI, Planejamento da Contratação, Seleção do Fornecedor e Gestão do Contrato. Na fase Gestão do Contrato são descritas as atividades e tarefas que devem ser realizadas durante a execução do contrato para que esse alcance os benefícios de negócio previstos. Dessa forma, os elementos desta fase contemplam o início, monitoramento e fim da contratação.

O Guia Prático de Contratações de TI [BRASIL 2011], publicado pelo MPOG, apresenta cinco processos e atividades para a fase de Gestão do Contrato de Solução de TI. Os processos possuem como intuito acompanhar e garantir o fornecimento dos bens e a adequada prestação dos serviços que compõem a solução de TI durante o período de execução do contrato.

Observa-se que há uma relação entre os elementos de gestão de contrato nas publicações supracitadas, sendo esses elementos ora sobrepostos, ora subdivididos em outros elementos conforme pode ser visto na Tabela 1.

Além dessa relação, é possível mapear o detalhamento dos elementos da gestão de contrato nas publicações. As Figuras 1 e 2 apresentam as fases da gestão do contrato segundo a IN04/2014. Para cada uma delas são relacionados os processos do Guia do TCU e as atividades do PCSTI. 
Tabela 1. Elementos comuns à gestão de contratos

\begin{tabular}{|c|c|c|c|}
\hline $\begin{array}{l}\text { Gestão do Contrato na } \\
\text { IN04/2014 }\end{array}$ & $\begin{array}{l}\text { Subfases } \\
\text { QRN }\end{array}$ & $\begin{array}{c}\text { Processos } \\
\text { Guia do TCU }\end{array}$ & $\begin{array}{l}\text { Atividades } \\
\text { PCSTI }\end{array}$ \\
\hline Início do contrato & $\begin{array}{l}\text { Iniciação do } \\
\text { contrato }\end{array}$ & Iniciação & Iniciar o contrato \\
\hline $\begin{array}{l}\text { Encaminhamento } \\
\text { formal de demandas }\end{array}$ & \multirow{5}{*}{$\begin{array}{l}\text { Execução do } \\
\text { contrato }\end{array}$} & $\begin{array}{c}\text { Encaminhar } \\
\text { ordem de serviço }\end{array}$ & Encaminhar demandas \\
\hline \multirow{4}{*}{$\begin{array}{l}\text { Monitoramento da } \\
\text { execução }\end{array}$} & & \multirow{4}{*}{$\begin{array}{l}\text { Monitoramento } \\
\text { da execução }\end{array}$} & $\begin{array}{c}\text { Realizar o monitoramento } \\
\text { técnico }\end{array}$ \\
\hline & & & Executar a atestação técnica \\
\hline & & & $\begin{array}{c}\text { Realizar o monitoramento } \\
\text { administrativo }\end{array}$ \\
\hline & & & $\begin{array}{l}\text { Tratar as demandas por } \\
\text { alterações contratuais }\end{array}$ \\
\hline $\begin{array}{l}\text { Transição e } \\
\text { Encerramento } \\
\text { contratual }\end{array}$ & $\begin{array}{l}\text { Encerramento } \\
\text { do contrato }\end{array}$ & $\begin{array}{c}\text { Transição } \\
\text { contratual e } \\
\text { Encerramento do } \\
\text { contrato }\end{array}$ & $\begin{array}{l}\text { Realizar o encerramento } \\
\text { contratual e a transição }\end{array}$ \\
\hline
\end{tabular}

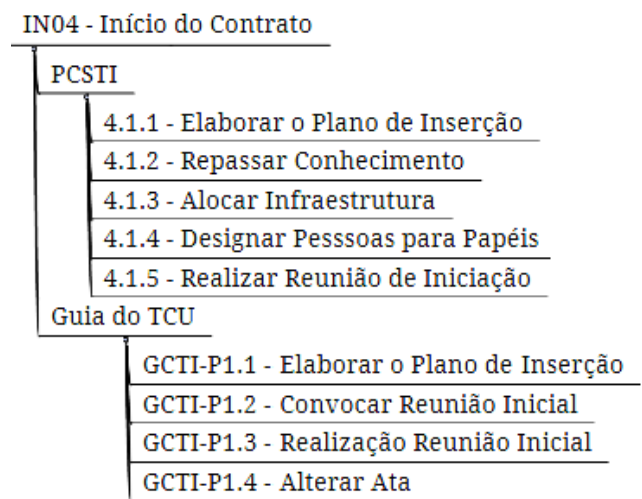

IN04 - Encaminhamento Formal de Demandas
IN04 - Transição e Encerramento Contratual

\begin{tabular}{|c|c|}
\hline \multicolumn{2}{|c|}{ PCTSI } \\
\hline \multicolumn{2}{|r|}{$\begin{array}{l}\text { 4.7.1 Realizar procedimento de } \\
\text { transferência de conhecimento }\end{array}$} \\
\hline & $\begin{array}{l}\text { 4.7.2 Realizar procedimento de } \\
\text { transferência de recurso }\end{array}$ \\
\hline & $\begin{array}{l}\text { 4.7.3 Realizar procedimento de } \\
\text { segurança da informação }\end{array}$ \\
\hline & $\begin{array}{l}\text { 4.7.4 Verificar a quitação de encargos } \\
\text { trabalhistas e previdenciais }\end{array}$ \\
\hline \multicolumn{2}{|r|}{ Guia do TCU } \\
\hline & GCTI-P4 - Transição Contratual \\
\hline & $\begin{array}{l}\text { GCTI-P5 - Recuperação de Recursos } \\
\text { de Propriedade da Contratada }\end{array}$ \\
\hline & GCTI-P5 - Remoção de Acesso \\
\hline
\end{tabular}

\begin{tabular}{|l}
$\frac{\text { PCSTI }}{4.2 .1}$ - Receber Demanda de Negócio \\
$\frac{4.2 .2 \text { - Elaborar Ordem de Serviço }}{4.2 .3 \text { - Assinar Ordem de Serviço }}$ \\
Guia do TCU \\
GCTI-P2 - Encaminhar Ordem de \\
Serviço
\end{tabular}

Figura 1. Relação dos Elementos de Gestão do Contrato - Iniciação, Encaminhamento Formal de Demandas e Transição e Encerramento Contratual

\section{Metodologia}

O desenvolvimento deste trabalho foi constituído de quatro fases: Desenvolvimento do checklist, Seleção das ferramentas, Avaliação das ferramentas e Análise e Redação dos resultados. 
Para o Desenvolvimento do checklist foi realizado um estudo sobre as normas e modelos vigentes para a contratação de soluções de TI em órgãos públicos, a fim de entender as necessidades de suporte para a gestão do contrato que deveriam ser contempladas por uma ferramenta de gestão de contratos de serviços de TI.

Para a Seleção das Ferramentas buscou-se extrair a estrutura de categorização de software no PSBP, para a seleção das ferramentas que se propunham a apoiar a gestão de contratos.

Para a Avaliação das Ferramentas, o checklist desenvolvido foi aplicado a cada ferramenta selecionada.

Para a Análise e Redação dos Resultados foram analisadas e descritas as ferramentas quanto à aderência aos elementos de gestão de contrato de serviços de TI para órgãos públicos brasileiros.

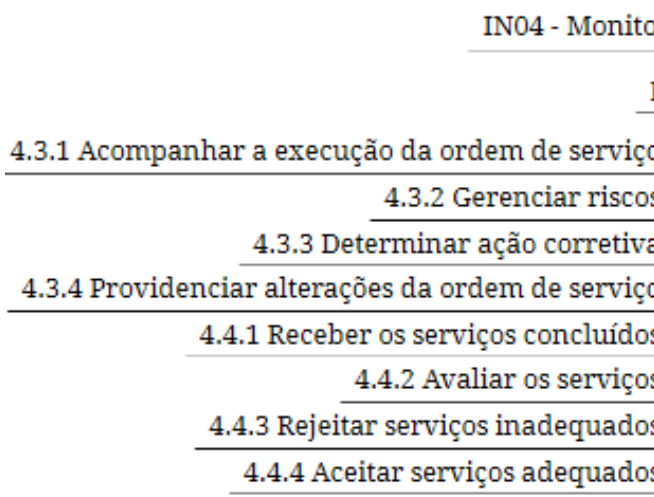
4.5.1 Acompanhar os Serviços em Execução

4.5.2 Avaliar cumprimento do contrato 4.5.3 Aplicar sanções

4.5.4 Corrigir desatendimento contratual 4.5.5 Calcular valores dos serviços e impostos 4.5.6 Liquidar a despesa

4.5.7 Autorizar emissão de fatura ou nota fiscal 4.5.8 Verificar regularidade fiscal, comercial, trabalhista e previdenciária

4.5.9 Efetuar pagamento dos serviços 4.6.1 Tratar alteração de quantitativos

4.6.2 Tratar alteração de prazos de execução

4.6.3 Tratar alteração de cláusulas contratuais 4.6.4 Tratar alteração técnica 4.6.5 Tratar readequação de preços 4.6.6 Tratar prorrogação do contrato 4.6.7 Tratar suspensão de contrato 4.6.8 Tratar rescisão de contrato

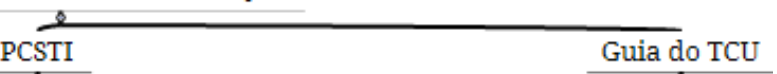

\section{I}

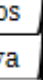
.

\section{.} .

\section{.}

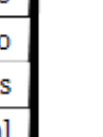

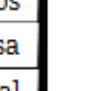

$$
\text { , }
$$

$$
\text { 帛 }
$$$$
\text { . }
$$

$$
\text { S }
$$

$$
\text { atis }
$$

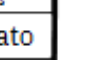

\section{GCTI-P3.1 - Receber Objeto \\ GCTI-P3.2 - Elaborar Termo de Recebimento Provisório}

GCTI-P3.3 - Avaliar Qualidade

GCTI-P3.4 - Analisar Desvios de Qualidade

GCTI-P3.5 - Encaminhar

Demandas de Correção

GCTI-P3.6 - Efetuar Correções

GCTI-P3.7 - Verificar Aderência

aos Termos Contratuais

GCTI-P3.8 - Indicar Termos Não Aderentes

GCTI-P3.9 - Encaminhar

Sanções Para Área

Administrativa

GCTI-P3.10 - Elaborar Termo de

Recebimento Definitivo

GCTI-P3.11 - Autorizar Emissão de Nota

Fiscal

GCTI-P3.12 - Emitir Nota Fiscal

GCTI-P3.13 - Verificar

Irregularidades Fiscais,

Trabalhistas e Previdenciárias

GCTI-P3.14 - Verificar

Manutenção da Necessidade,

Economicidade e Oportunidade

GCTI-P3.15 - Encaminhar

Pedido de Alteração Contratual

Figura 2. Relação dos Elementos de Gestão do Contrato - Monitoramento da Execução 


\section{Desenvolvimento do Checklist}

A partir dos estudos sobre a IN 04 [BRASIL 2014], do PCSTI [CRUZ, ANDRADE E FIGUEIREDO 2010], e do Guia de Boas Práticas em Contratação de Solução de TI [BRASIL 2012], foram elencados elementos de gestão do contrato previstos nessas fontes.

Os elementos elencados, que estão alinhados à legislação vigente brasileira, foram identificados e organizados em Características Essenciais e em Características Desejáveis que uma ferramenta de gestão deveria contemplar para possibilitar que a gestão do contrato seja realizada alinhada às diretrizes preconizadas por essas fontes.

Os elementos considerados essenciais estão relacionados ao gerenciamento do início do contrato, dos planos contratuais, das ordens de serviço, da execução do serviço, dos riscos, dos custos e dos elementos do contrato, conforme apresentado na Tabela 2.

Tabela 2. Elementos que compõem as Características Essenciais

\begin{tabular}{|c|c|c|c|c|}
\hline Característica & Suporte & IN04/2014 & PCSTI & Guia \\
\hline $\begin{array}{l}\text { Gerenciamento do Início do } \\
\text { Contrato }\end{array}$ & Definição de perfil & Art. 32 , item I & 4.1 .1 & GCTI-P1.1 \\
\hline \multirow{3}{*}{$\begin{array}{l}\text { Gerenciamento dos Planos } \\
\text { Contratuais }\end{array}$} & Plano de Inserção & Art. 32 , item I & 4.1 .1 & GCTI-P1.1 \\
\hline & Plano de Fiscalização & Art. 32 , item II & - & - \\
\hline & Plano de Sustentação & - & $\begin{array}{c}4.7 .1,4.7 .2 \mathrm{e} \\
4.7 .3 \\
\end{array}$ & GCTI-P4 \\
\hline \multirow{3}{*}{$\begin{array}{c}\text { Gerenciamento das Ordens de } \\
\text { Serviço }\end{array}$} & Registro da ordem de serviço & Art. 35 , item I & 4.2 .2 & - \\
\hline & Atualização da ordem de serviço & \multirow{2}{*}{ Art 34 , item I } & \multirow{2}{*}{4.3 .4} & - \\
\hline & Histórico de atualização & & & - \\
\hline \multirow{8}{*}{$\begin{array}{l}\text { Gerenciamento da Execução } \\
\text { do Serviço }\end{array}$} & Registro do Termo de Recebimento & Art. 34, item I & 4.4 .4 & GCTI-P3.2 \\
\hline & Registro do Termo de Recebimento & Art. 34, item & 4.5 .2 & GCTI-P3.10 \\
\hline & Registro de não conformidades contratuais & Art. 34, item III & 4.3 .1 & GCTI-P3.5 \\
\hline & Acompanhamento dos SLAs & - & 4.4 .2 e 4.4 .3 & $\begin{array}{l}\text { GCTI-P3.6 } \\
\text { GCTI-P3.8 }\end{array}$ \\
\hline & Relatório de avaliação dos serviços & Art. 34 , item II & 4.4 .2 & $\begin{array}{l}\text { GCTI-P3.3 } \\
\text { GCTI-P3.4 }\end{array}$ \\
\hline & Registro de sanções e glosas & Art. 20, item III & $4.3 .3 \mathrm{e} 4.5 .3$ & GCTI-P3.9 \\
\hline & Registro do diário do contrato & Art. 34, item & $4.5 .1,4.2 .1$ & - \\
\hline & Registro da alocação de recursos & Art. 2, item XVI & 4.1 .3 & - \\
\hline Gerenciamento dos Riscos & Registro do Relatório dos riscos & \begin{tabular}{|c|} 
Art. 2 , item XV \\
Art. 13 \\
\end{tabular} & 4.3 .2 & - \\
\hline Gerenciamento dos Custos & Registro das notas fiscais e pagamentos & \begin{tabular}{|l|} 
Art. 20 , item V \\
Art. 34, item IX \\
\end{tabular} & $\begin{array}{r}4.5 .5,4.5 .6 \\
4.5 .7 \text { e } 4.5 .9 \\
\end{array}$ & $\begin{array}{l}\text { GCTI-P3.11 } \\
\text { GCTI-P3.12 } \\
\end{array}$ \\
\hline \multirow{2}{*}{$\begin{array}{c}\text { Gerenciamento } \\
\text { dos Elementos do Contrato }\end{array}$} & $\begin{array}{l}\text { Acompanhamento dos quantitativos, prazos, } \\
\text { claúsulas e preços. }\end{array}$ & \begin{tabular}{|c|} 
Art. 19 \\
Art. 34, item \\
\end{tabular} & $\begin{array}{c}4.6 .1,4.6 .2 \\
4.6 .3\end{array}$ & GCTI-P3.15 \\
\hline & $\begin{array}{l}\text { Registro da Prorrogações, Suspensão e } \\
\text { Rescisão }\end{array}$ & - & $\begin{array}{c}4.6 .6,4.6 .7 \mathrm{e} \\
4.6 .8\end{array}$ & - \\
\hline
\end{tabular}

Os elementos considerados desejáveis são relacionados às características de gerenciamento de reuniões, de demandas e do encerramento do contrato, e abordam apoios adicionais para o gerenciamento das ordens de serviço e da execução do serviço, conforme apresentado na Tabela 3.

Os elementos caracterizados como essenciais e desejáveis apresentados nas Tabelas 2 e 3 são correlacionados ao detalhamento apresentado nas Figuras 1 e 2, onde um elemento refere-se a um identificador. 
Tabela 3. Elementos que compõem as Características Desejáveis

\begin{tabular}{|c|c|c|c|c|}
\hline Característica & Suporte & IN04/2014 & PCSTI & Guia \\
\hline \multirow{2}{*}{$\begin{array}{c}\text { Gerenciamento das Ordens de } \\
\text { Serviço }\end{array}$} & Armazenamento da ordem assinada & $\begin{array}{l}\text { Art. 33, item I } \\
\text { Art. 33, item II }\end{array}$ & 4.2 .3 & - \\
\hline & Registro da conclusão da ordem de serviço & - & 4.4 .1 & - \\
\hline Gerenciamento de Reuniões & Registro de atas & Art. 32, item III & 4.1 .5 & GCTI-P1.4 \\
\hline \multirow{2}{*}{ Gerenciamento de Demandas } & Registro de demandas & - & 4.2 .1 & - \\
\hline & Priorização de demandas & - & 4.2 .1 & - \\
\hline \multirow{3}{*}{$\begin{array}{c}\text { Gerenciamento da Execução } \\
\text { do Serviço }\end{array}$} & $\begin{array}{l}\text { Registro de correção dos serviços à } \\
\text { contratada }\end{array}$ & \begin{tabular}{|l|} 
Art. 34, item III \\
Art. 34, item IV \\
\end{tabular} & - & GCTI-P3.5 \\
\hline & Registro do Termo de Rejeição & - & 4.4 .3 & - \\
\hline & Registro do Termo de Aceite & - & 4.4 .4 & - \\
\hline $\begin{array}{c}\text { Gerenciamento do } \\
\text { Encerramento do Contrato }\end{array}$ & Termo de Encerramento do Contrato & - & - & GCTI-P5 \\
\hline
\end{tabular}

Utilizando como base os elementos identificados, foram estabelecidos dois checklists, um para as características essenciais e outro para as desejáveis. Estes checklists foram utilizados para realizar as avaliações das ferramentas, apresentadas na Seção 5.2 - Avaliação das Ferramentas a partir do Checklist.

Nas subseções seguintes apresentam-se os itens que compõem os Checklist de elementos essenciais e de elementos desejáveis.

\subsection{Checklist de Elementos Essenciais}

Apresentam-se os itens que compõem o checklist Essencial:

- Gerenciamento do Início do Contrato

1) Controla os níveis de acesso os quais podem realizar alterações nos planos?

- Gerenciamento de Planos Contratuais

2) Dispõe de mecanismos para gerenciamento do plano de Inserção?

3) Dispõe de mecanismos para gerenciamento do plano de Fiscalização?

4) Dispõe de mecanismos para gerenciamento do plano de Sustentação?

5) Registra alterações nos planos acima mencionados, bem como o demandante da alteração?

- Gerenciamento das Ordens de Serviços

6) Dispõe do aparato para o registro das ordens de serviços referentes ao contrato?

7) Permite o gerenciamento das ordens de serviço?

8) Possibilita a visualização das alterações, bem como seus demandantes?

- Gerenciamento da Execução do Serviço

9) Dispõe de mecanismo para gerenciamento do termo de Recebimento Provisório?

10) Dispõe de mecanismo para gerenciamento do termo de Recebimento Definitivo? 
11) Dispõe do aparato para o registro de Não Conformidades Contratuais?

12) Possibilita o gerenciamento dos Níveis Mínimos de Serviços (SLA), bem como a geração de relatório sobre?

13) Habilita a avaliação dos serviços ou registro do relatório de avaliação dos serviços?

14) Dispõe de um procedimento para aplicação/registro de sanções e glosas contratuais?

15) Dispõe de mecanismos para registro do diário do contrato?

16) Permite a alocação e registro de recursos para o contrato?

- Gerenciamento de Riscos

17) Dispõe de mecanismos para registro dos riscos contratuais, bem como suas ações de mitigação?

18) Permite avaliar os riscos e classificá-los de acordo com sua severidade?

- Gerenciamento de Custos

19) Dispõe de mecanismos para registrar notas fiscais e pagamentos realizados?

20) Dispõe de mecanismos para registrar os custos dos serviços e impostos?

- Gerenciamento dos Elementos do Contrato

21) Dispõe de mecanismos para gerenciamento dos quantitativos, prazos, cláusulas e preços do contrato?

22) Possibilita registrar ações de prorrogações, suspensões e/ou rescisões contratuais, bem como gerenciar os registros destas ações?

\subsection{Checklist de Elementos Desejáveis}

Apresentam-se os itens que compõem o checklist Desejável:

- Gerenciamento das Ordens de Serviços

1) Possibilita o armazenamento das ordens de serviço assinadas?

2) Possibilita registrar a conclusão de uma ordem de serviço?

- Gerenciamento de Reuniões

3) Dispõe de mecanismo para gerenciamento das atas de reunião?

- Gerenciamento de Demandas

4) Dispõe do aparato para o registro de demandas do contrato?

5) Dispõe de um mecanismo para a priorização das demandas do contrato?

- Gerenciamento da Execução do Serviço

6) Possibilita registrar as correções necessárias aos serviços prestados?

7) Dispõe de mecanismos para gerenciamento do termo de rejeição?

8) Dispõe de mecanismos para gerenciamento do termo de aceite? 
- Gerenciamento do Encerramento do Contrato

9) Dispõe de mecanismos para gerenciamento do termo de encerramento do contrato?

Com o desenvolvimento do checklist a partir das fontes selecionadas, buscou-se selecionar as ferramentas disponíveis no PSPB.

\section{Seleção das Ferramentas}

O Portal do Software Público Brasileiro (PSPB) possui atualmente 72 soluções de software em seu catálogo, dispostos em 26 categorias, de diversas áreas de negócio.

\subsection{Seleção no PSPB}

Para selecionar as ferramentas no PSPB, utilizou-se o filtro de categorias, sendo que a categoria inicialmente analisada foi a de "Planejamento e Gestão". Para seleção das ferramentas foi analisado o título e sua descrição disponível no PSPB. Ao todo, foram selecionadas apenas três ferramentas nesta categoria, apresentadas na Tabela 4.

Tabela 4. Ferramentas selecionadas no PSPB

\begin{tabular}{|c|c|c|}
\hline Categorias do PSPB & Ferramentas & Resultante da avaliação \\
\hline Comunicações & OASIS & Descartada por falta de aderência \\
\hline Comunicações & Citsmart-ITSM & Avaliada nos dois checklists \\
\hline Administração & SIMEC & Descartada por falta de aderência \\
\hline $\begin{array}{c}\text { Administração; } \\
\text { Planejamento e Gestão }\end{array}$ & Geplanes & Descartada por falta de aderência \\
\hline $\begin{array}{c}\text { Administração; } \\
\text { Planejamento e Gestão }\end{array}$ & Gepnet & Avaliada nos dois checklists \\
\hline $\begin{array}{c}\text { Administração; } \\
\text { Comunicação; Planejamento } \\
\text { e Gestão }\end{array}$ & GPWeb & Descartada por falta de aderência \\
\hline $\begin{array}{c}\text { Administração; } \\
\text { Economia e Finanças }\end{array}$ & Urbem CNM & Descartada por falta de aderência \\
\hline
\end{tabular}

Dado o número reduzido de ferramentas selecionadas, buscou-se por outras ferramentas em categorias que se relacionavam com as ferramentas selecionadas. As outras categorias foram "Administração" e "Comunicações". Após a inclusão das categorias mencionadas para a seleção das ferramentas, o número de ferramentas encontradas aumentou para sete, conforme apresentado na Tabela 4.

\subsection{Avaliação das Ferramentas}

Para avaliação das ferramentas foram separadas e analisadas as documentações disponíveis - especificação de requisitos, wiki, documento de negócio, entre outros - das ferramentas selecionadas, no PSPB ou em suas homepages. Em um segundo momento, as ferramentas foram instaladas e utilizadas pelos autores. Com a utilização das 
ferramentas e consultas aos manuais e/ou documentações disponíveis, as ferramentas foram avaliadas conforme itens do checklist definidos.

Para o critério de aderência ou não aderência, as ferramentas foram avaliadas em relação ao conteúdo do checklist essencial e, caso não atingisse pelo menos $50 \%$ de aderência no checklist essencial, a ferramenta seria dada como descartada. Se possuísse pelo menos $50 \%$ de aderência, seria avaliada nos elementos desejáveis.

A ferramenta OASIS $^{1}$ permite o acompanhamento dos projetos desenvolvidos pelas áreas de TI e dos contratos de empresas terceirizadas. Embora na descrição da ferramenta esteja contemplado o acompanhamento do contrato, a ferramenta não contempla muitos dos itens do checklist, atingindo apenas 18,2\%. A ferramenta pode ser caracterizada como uma ferramenta de suporte ao planejamento da contratação de serviços de TI.

A ferramenta Citsmart ${ }^{2}$ é uma solução multiplataforma de gerenciamento de serviços de TI. É dividida em três módulos de gerenciamento, sendo que um deles trata do ciclo de vida da gestão de contratos. A ferramenta obteve mais de $50 \%$ de aderência aos elementos propostos.

A ferramenta $\operatorname{SIMEC}^{3}$ é um software de gestão integrada de funções administrativas com destaque para programação orçamentária e gestão de projetos, desenvolvido pelo Ministério da Educação. A ferramenta atingiu 36,3\% de aderência aos elementos essenciais, e foi descartada.

A ferramenta Geplanes ${ }^{4}$ apoia a gestão estratégica de empresas públicas ou privadas. Pela descrição da ferramenta e, posteriormente, pela aplicação do checklist, a ferramenta não contempla a gestão de contratos de TI de uma empresa, e sim, para a gestão do planejamento estratégico em nível organizacional. A ferramenta atingiu apenas $18,2 \%$ de aderência aos elementos essenciais, e foi descartada.

A ferramenta GEPNET ${ }^{5}$ é uma solução desenvolvida para gestão de escritórios e de projetos. Permite o acompanhamento dos projetos da organização e o registro do ciclo de vida dos projetos com base nos processos do guia PMBOK (PMI). A ferramenta contemplou a maior parte dos itens do checklist essencial. Com isso, a ferramenta passa para a próxima etapa - análise de aderência aos elementos do checklist desejáveis.

A ferramenta $\mathrm{GPWeb}^{6}$ trata da gestão estratégica e do gerenciamento de projetos. A ferramenta foi avaliada em relação aos itens essenciais à uma ferramenta de apoio à gestão contratual e não atingiu metade de aderência ao checklist. Portanto, foi descartada.

\footnotetext{
${ }^{1}$ Oasis - Disponível em: https://softwarepublico.gov.br/social/oasis

${ }^{2}$ Cistmart IT - Service Deck - Disponível em: http://www.citsmart.com.br

${ }^{3}$ SIMEC - Disponível em: https://softwarepublico.gov.br/social/simec

${ }^{4}$ Geplanes - Disponível em: http://geplanes.com.br/

${ }^{5}$ GEPNET - Disponível em: https://softwarepublico.gov.br/social/gepnet

${ }^{6}$ GPWeb - Disponível em: https://softwarepublico.gov.br/social/gpweb
} 
A ferramenta $\mathrm{URBEM}^{7}$ possui como objetivo o aperfeiçoamento da gestão pública. Atingiu apenas $17,4 \%$ de aderência ao checklist essencial, e foi descartada.

A avaliação das ferramentas que não foram descartadas, para o checklist de elementos essenciais pode ser vista na Tabela 5. E as ferramentas que prosseguiram e foram avaliadas quanto aos elementos desejáveis pode ser vista na Tabela 6 , nas quais as marcações representam a resposta "Sim".

Tabela 5. Avaliação das ferramentas quanto aos elementos essenciais

\begin{tabular}{|c|c|c|}
\hline \multirow{2}{*}{ Item do Checklist } & \multicolumn{2}{|c|}{ Ferramenta } \\
\hline & CitSmart & GEPNET2 \\
\hline 1 & $\mathrm{X}$ & $\mathrm{X}$ \\
\hline 2 & & \\
\hline 3 & & \\
\hline 4 & & $\mathrm{X}$ \\
\hline 5 & & \\
\hline 6 & $\mathrm{X}$ & $\mathrm{X}$ \\
\hline 7 & $\mathrm{X}$ & $\mathrm{X}$ \\
\hline 8 & $\mathrm{X}$ & \\
\hline 9 & $\mathrm{X}$ & $\mathrm{X}$ \\
\hline 10 & $\mathrm{X}$ & $\mathrm{X}$ \\
\hline 11 & $\mathrm{X}$ & $\mathrm{X}$ \\
\hline 12 & $\mathrm{X}$ & \\
\hline 13 & $\mathrm{X}$ & \\
\hline 14 & & \\
\hline 15 & $\mathrm{X}$ & \\
\hline 16 & $\mathrm{X}$ & $\mathrm{X}$ \\
\hline 17 & $\mathrm{X}$ & $\mathrm{X}$ \\
\hline 18 & $\mathrm{X}$ & $\mathrm{X}$ \\
\hline 19 & & \\
\hline 20 & & \\
\hline 21 & $\mathrm{X}$ & $X$ \\
\hline 22 & $\mathrm{X}$ & \\
\hline
\end{tabular}

Tabela 6. Avaliação das ferramentas para os elementos desejáveis

\begin{tabular}{|c|c|c|}
\hline \multirow{2}{*}{ Item do Checklist } & CitSmart & Ferramenta \\
\cline { 2 - 3 } & $\mathrm{X}$ & $\mathrm{X}$ \\
\hline 1 & $\mathrm{X}$ & $\mathrm{X}$ \\
\hline 2 & & $\mathrm{X}$ \\
\hline 3 & $\mathrm{X}$ & $\mathrm{X}$ \\
\hline 4 & & $\mathrm{X}$ \\
\hline 5 & $\mathrm{X}$ & $\mathrm{X}$ \\
\hline 6 & & \\
\hline 7 & & \\
\hline 8 & & \\
\hline 9 & & \\
\hline
\end{tabular}

${ }^{7}$ URBEM - Disponível em: https://softwarepublico.gov.br/social/urbem 
Os itens, Mecanismos para priorização das demandas do contrato e de gerenciamento do termo de rejeição, não foram contemplados por nenhuma das ferramentas.

Para os elementos desejáveis avaliados na Tabela 6 , a ferramenta GEPNET teve uma aderência de $66,6 \%$ e a CitSmart de $44,4 \%$ em relação aos elementos do ckecklist. Assim, como para os elementos essenciais, alguns elementos desejáveis foram contemplados por ambas as ferramentas e outros por apenas uma delas.

\section{Análise dos Resultados}

Considerando a avaliação apresentada na Tabela 5, que trata dos elementos essenciais, observa-se que a ferramenta mais aderente aos itens avaliados foi a ferramenta CitSmart, com uma aderência de $68,2 \%$, seguida pela ferramenta GEPNET, que teve uma aderência de 50\%.

Alguns elementos foram contemplados por ambas as ferramentas e outros por apenas uma delas. Ambas as ferramentas não possuem suporte específico para os planos de inserção e de fiscalização, para aplicação/registro de sanções e glosas contratuais e para o gerenciamento de custo.

Para os elementos desejáveis avaliados na Tabela 6, a ferramenta GEPNET teve uma aderência de $66,6 \%$ e a CitSmart de 44,4\%. Assim como para os elementos essenciais, alguns elementos desejáveis foram contemplados por ambas as ferramentas e outros por apenas uma delas. Os elementos, Mecanismos para priorização das demandas do contrato e de gerenciamento do termo de rejeição, não foram contemplados por nenhuma das ferramentas.

\section{Considerações Finais}

Com as revisões bibliográfica e documental foi possível selecionar elementos essenciais e desejáveis para a construção de um instrumento de checklist de ferramentas que deveriam dar suporte à gestão de contratos prevista na IN 04, no Guia de Contratações de Soluções de TI e no PCSTI.

Com base na avaliação das ferramentas selecionadas do PSPB, foi possível observar que os suportes esperados, identificados a partir do checklist essencial, possuem uma baixa aderência por parte das ferramentas. E que somente alguns dos suportes desejáveis foram atendidos. Uma das razões para a baixa aderência está relacionada ao fato de que as ferramentas disponíveis não têm como escopo principal realizar a gestão do contrato.

Com o produto dessa pesquisa, um checklist disponível a comunidade, os órgãos podem justificar o desenvolvimento de uma ferramenta de gestão de contratos, pois não há no PSPB até o momento desta pesquisa, uma ferramenta de gestão de contratos de serviços de TI aderente à legislação brasileira.

$\mathrm{O}$ checklist produzido atende às expectativas propostas a partir do objetivo deste trabalho. Dando sequência a este trabalho, vislumbra-se o desenvolvimento de uma ferramenta de gestão de contratos de serviços de TI pelos órgãos públicos brasileiros, que atenda aos elementos esperados segundo os checklists desenvolvidos de elementos essenciais e desejáveis neste trabalho. 


\section{Referências}

Brasil (1993), Presidência da República, Casa Civil. Lei n 8.666/1993. Regulamenta o art. 37, inciso XXI, da Constituição Federal, institui normas para licitações e contratos da Administração Pública e dá outras providências. 1993.

Brasil (1967), Presidência da República, Casa Civil. Decreto-Lei no 200/1967. Dispõe sobre a organização da Administração Federal, estabelece diretrizes para a Reforma Administrativa e dá outras providências. 1967.

Brasil (2003), Tribunal de Contas da União. Acórdão 1.558/2003-TCU-Plenário. Auditoria. Coordenação-Geral de Serviços Gerais do Ministério do Desenvolvimento, Indústria e Comércio Exterior. Área de aquisições de bens e serviços de informática. Processo de denúncia apensado para apuração conjunta. 2003. BRASIL, Tribunal de Contas da União.

Brasil (2008), Tribunal de Contas da União. Acórdão 1.603/2008-Plenário TCU. Auditoria. Coordenação-Geral de Serviços Gerais do Ministério do Desenvolvimento, Indústria e Comércio Exterior. Área de aquisições de bens e serviços de informática. Processo de denúncia apensado para apuração conjunta. 2003. BRASIL, Tribunal de Contas da União. Acórdão 786/2006-TCU-Plenário. 2006./acd786_06.pdf

Brasil (2009), Tribunal de Contas da União. Acórdão 1.215/2009-TCU-Plenário. Relatório de auditoria. Apartado. Estudo sobre os parâmetros balizadores da contratação de serviços de TI. Elaboração de Quadro Referencial Normativo (QRN).

Brasil (2011), Ministério do Planejamento, Orçamento e Gestão. Guia Prático para contratação de soluções de tecnologia da informação, 2011. Disponível em: http://www.ifmg.edu.br/downloads/guia-pratico-para-contratacao-de-solucoes-de-tiv1.1.pdf

Brasil (2012), Tribunal de Contas da União. Guia de Boas Práticas em Contratação de Soluções de Tecnologia da Informação, 2012. Disponível em: http://portal2.tcu.gov.br/portal/pls/portal/docs/2511467.PDF

Brasil (2014), Ministério do Planejamento, Orçamento e Gestão. Instrução Normativa MP/SLTI No 4, de 11 de setembro de 2014, 2014. Disponível em: $\mathrm{http}: / / \mathrm{www}$.governoeletronico.gov.br/sisp-conteudo/nucleo-de-contratacoes-deti/modelo-de-contratacoes-normativos-e-documentos-de-referencia/instrucaonormativa-mp-slti-no04

Brasil (2016). Ministério do Planejamento Orçamento e Gestão, Secretaria de Tecnologia da Informação. Portaria MP/STI n 20 (2016). Disponível em http://www.governoeletronico.gov.br/biblioteca/arquivos/portaria-mp-sti-no-20-de14-de-junho-de-2016/download

Brasil (2016). Ministério do Planejamento, Desenvolvimento e Gestão. Portal do Software Público Brasileiro (2016). Disponível em: https://softwarepublico.gov.br/social/spb/sobreoportal

Cruz, C. S. (2008), "Governança de TI e conformidade legal no setor público: um quadro referencial normativo para a contratação de serviços de TI", Dissertação, Universidade Católica de Brasília. 2008. 
Cruz, C. S., Andrade, E. P., Figueiredo, R. M. C., (2010), "Processo de Contratação de Serviços de Tecnologia da Informação para Organizações Públicas", Ministério da Ciência e Tecnologia. Secretaria de Política de Informática, Brasília, Brasil, 2010.

Daud Júnior, A., Vaz, W, (2016), A Descomplicada Contratação de TI na Administração Pública. Tangente Editora. 